\title{
Effects of alloy disorder and confinement on phonon modes and Raman scattering in $\mathrm{Si}_{\mathrm{x}} \mathrm{Ge}_{1-\mathrm{x}}$ nanocrystals: a microscopic modeling
}

\author{
A. S. Vasin ${ }^{a}$, O. V. Vikhrova ${ }^{a}$, M. I. Vasilevskiy \\ ${ }^{a}$ Department of Physics and NIFTI, N. I. Lobachevskii University of Nizhnii \\ Novgorod, Nizhnii Novgorod 603600, Russia \\ ${ }^{\mathrm{b}}$ Centro de Física, Universidade do Minho, Campus de Gualtar, 4710-057 Braga, \\ Portugal
}

Corresponding author: mikhail@fisica.uminho.pt

PACS numbers: 63.50.Gh, 63.10.+a, 63.20.Pw, 78.67.-n

Keywords: nanocrystal, alloying, phonon, Raman scattering

Confinement and alloy disorder effects on the lattice dynamics and Raman scattering in $\mathrm{Si}_{1-x} \mathrm{Ge}_{x}$ nanocrystals (NCs) are investigated numerically employing two different empirical inter-atomic potentials. Relaxed NCs of different composition $(x)$ were built using the Molecular Dynamics method and applying rigid boundary conditions mimicking the effect of surrounding matrix. The resulting variation of bond lengths with $x$ was checked against Vegard's law and the NC phonon modes were calculated using the same inter-atomic potential. The localization of the principal Raman-active ( $\mathrm{Si}-\mathrm{Si}, \mathrm{Si}-\mathrm{Ge}$ and $\mathrm{Ge}-\mathrm{Ge}$ ) modes is investigated by analysing representative eigenvectors and their inverse participation ratio. The dependence of the position and intensity of these modes upon $x$ and NC size is presented and compared to previous calculated results and available experimental data. In particular, it is argued that the composition dependence of the intensity of the Si-Ge and Ge-Ge modes does not follow the fraction of the corresponding nearest-neighbour bonds as it was suggested by some authors. Possible effects of alloy segregation are considered by comparing the calculated properties of random and clustered $\mathrm{Si}_{x} \mathrm{Ge}_{1-x} \mathrm{NCs}$. It is found that the Si$\mathrm{Si}$ mode and Ge-Ge modes are enhanced and blue-shifted (by several $\mathrm{cm}^{-1}$ for the $\mathrm{Si}-\mathrm{Si}$ mode), while the intensity of the Si-Ge Raman mode is strongly suppressed by clustering. 


\section{Introduction}

Crystalline silicon (Si), the most important semiconductor material in microelectronics and energy conversion technologies, would be suitable also for optoelectronics if it were possible to bypass the shortcoming related to its indirect band gap. The possibility of obtaining the desired band structure with a controlled gap in $\mathrm{Si}$ nanocrystals (NCs) is still considered as a plausible solution [1]. Adding germanium $(\mathrm{Ge})$ provides an additional means of adjusting the properties of the NCs, in terms of e.g. optical gap or static dielectric constant, while this random alloy retains, on average, the diamond structure throughout the entire composition range. A variety of nearly zero dimensional $\mathrm{Si}-\mathrm{Ge}$ alloy nanostructures have been obtained and studied, including self-assembled quantum dots (QDs) [2-5], NCs embedded in $\mathrm{SiO}_{2}$ matrix [6-8] and even free-standing NCs [9]. It is expected that Si-Ge nanoparticles with well controlled size and composition will soon be synthesized by colloidal chemistry techniques in a manner similar to their II-VI counterparts [10]. The possibility of fabrication of $\mathrm{Si}-\mathrm{Ge}$ alloy nanoparticles by pyrolysis of silane and germane has also been demonstrated recently [11]. These methods can be elaborated further to make a step towards fabrication of group IV NC optoelectronic, thermoelectronic and photovoltaic devices.

Raman spectroscopy, non-destructive and sensitive, is a method of choice to investigate nanomaterials [12]. It was used in most of the above mentioned works on $\mathrm{Si}-\mathrm{Ge} \mathrm{NCs}$ and QDs [2-9], as well as it has been applied to $\mathrm{Si}_{x} \mathrm{Ge}_{1-x}$ alloys in bulk [13] and epitaxial layer [14] forms. Independently of crystal dimension, the alloy spectra are characterized by three major peaks centred near 300,400 and $500 \mathrm{~cm}^{-1}$, associated with optical vibrations involving $\mathrm{Ge}-\mathrm{Ge}, \mathrm{Si}-\mathrm{Ge}$, and $\mathrm{Si}-\mathrm{Si}$ stretching motions, respectively [13]. Smaller details of the spectra, such as precise positions of the main scattering bands, their broadening, and weaker peaks that have been observed in some of the studies depend on the preparation method, NC size, etc. Their assignment has attracted considerable attention over decades [15], however, it seems that additional theoretical and computational work is required for their correct interpretation in nanostructures. As for any confined system with alloy disorder, there are several effects that can be involved simultaneously, affecting its electronic and vibrational properties, such as non-local elastic strain [5], interplay between phonon confinement and (competing with it) localization owing to alloy disorder [16], formation of percolating clusters [15] or non-random distribution of two types of atoms in the alloy [17]. These effects should influence the dependence of the electronic energy levels and phonon modes upon the NC size and composition.

Several works have been performed, devoted to the calculation of phonon properties and Raman spectra of $\mathrm{Si}_{1-x} \mathrm{Ge}_{x}$ alloys [13-15,17-23], some of which considered nanostructures [15,17-22]. Because of the absence of translational symmetry, the use of more accurate $a b$ initio models is limited to small NCs $[15,17,18,20,21]$ or have to be combined with an approximation scheme [22], while empirical potential models used in $[13,14,18,19,23]$ can be applied to larger systems and produce results that are insightful for the understanding of the alloying and confinement effects in mixed NCs. We have reported some preliminary results on bond lengths and Raman spectra of Si-Ge alloy NCs [24], obtained by using the Tersoff semi-empirical potentials (TP) [25], that seem to be in quite a good agreement with experimental data.

In this work, we present the vibration properties of $\mathrm{Si}_{x} \mathrm{Ge}_{1-x}$ crystallites of different composition and size calculated using two different empirical inter-atomic potentials, TP and those proposed by Stillinger and Weber [26]. Both potentials take into account 
three-particle interactions, that is, depend on the bond angles whose variation is known to contribute to the strain relaxation in the alloy where the end members have different lattice constants [27]. They are often used for calculations of lattice properties of Si-Ge alloy crystals, e.g. phonon-related thermal conductivity [28]. The advantage of the Stillinger-Weber (SWP) and Tersoff potentials, in comparison with the widely used valence force field (VFF) model (employed in $[18,19,23]$ ), is that they can be used for building a crystallite by relaxing it to thermodynamic equilibrium (for a given composition), before considering the lattice vibrations, while the VFF model approach is limited to the harmonic approximation (eventually with anharmonic corrections [23]). Therefore, we are able to take into account the realistic three-modal distribution of bond lengths in these alloy NCs [24]. We shall present the density of phonon states (DS) and the inverse participation ratio (IPR) [29] for some characteristic vibration modes, as well as non-resonant Raman scattering spectra. Our goal is to investigate the interplay between phonon confinement inside NC and shortrange localization due to alloy disorder. Also, the effect of possible non-random distributions of $\mathrm{Si}$ and $\mathrm{Ge}$ atoms in the $\mathrm{NC}$ will be considered. In the next section we explain how relaxed $\mathrm{Si}_{x} \mathrm{Ge}_{1-x}$ alloy $\mathrm{NCs}$ are built and analyse the bond length distributions. In Sec. 3 we present and discuss DS, IPR and Raman spectra of the nanocrystals, calculated with TP and SWP for different NC size and composition. The last section is devoted to conclusions.

\section{Si-Ge NC's structure}

\subsection{Building relaxed Si-Ge crystallites}

$\mathrm{Si}_{1-x} \mathrm{Ge}_{x}$ crystallites of "approximately spherical" shape, containing up to $N=1647$ lattice sites were built by randomly distributing some $x N$ Ge and $(1-x) N \mathrm{Si}$ atoms over the sites of a diamond lattice, starting from a central atom and filling its 1-st, 2nd, ... coordination shells. Initially, the lattice constant was chosen according to Vegard's law,

$$
a=a_{S i} \cdot(1-x)+a_{G e} \cdot x,
$$

where $a_{S i}=0.543 \mathrm{~nm}$ and $a_{G e}=0.563 \mathrm{~nm}$ are the lattice constants of crystalline $\mathrm{Si}$ and Ge, respectively. These crystallites retained the $T_{d}$ symmetry with perfect tetrahedric bonding.

Interactions between the atoms were defined according to one of the empirical potentials (SWP or TP). Relaxation to the minimum of the total energy of the crystallite was achieved by allowing the atoms to move in response to the forces produced by their neighbours. Using the molecular dynamics (MD) method employing the Verlet algorithm [30] with integration of the equations of motion at each temporal step (not exceeding $10^{-15} \mathrm{~s}$ ) we obtained equilibrium crystallites that no longer possessed the $T_{d}$ symmetry (except for $x=0$ or 1 ).

Bearing in mind NCs embedded in a matrix, rigid boundary conditions were considered already at the relaxation stage, which means that the positions of the atoms in two outer shells were fixed, though they were considered interacting with the movable atoms of the interior shells. So, the movable atoms had all of their first and second nearest neighbors, which is necessary for the correct application of the SW 
and $\mathrm{T}$ potentials. The largest crystallites contained 981 movable atoms (corresponding to the NC diameter $D \approx 3.9 \mathrm{~nm}$ ).

\subsection{Bond length distribution}

The variation of the average bond lengths, for $\mathrm{Si}-\mathrm{Si}, \mathrm{Si}-\mathrm{Ge}$ and $\mathrm{Ge}-\mathrm{Ge}$ bonds, with alloy composition is shown in Fig. 1. There is some quantitative difference between the TP and SWP results, particularly for the mixed bonds, but qualitatively the bond length's dependence on $x$ is similar for the three types of bonds and for both potentials. It is much weaker than that predicted by Eq. (1), in agreement with the idea that the relaxation of the microscopic strain related to the difference between the bond lengths in pure $\mathrm{Si}$ and $\mathrm{Ge}$, occurs mostly via distortion of the bond angles and to a lesser extent by changing the bond lengths [31]. To quantify the contribution of these two mechanisms of strain relaxation, one can introduce so called topological rigidity parameter [27]:

$$
a_{i j}^{* * *}=1-\frac{\Delta_{i j}}{R_{G e}^{0}-R_{S i}^{0}}
$$

where $i, j=\mathrm{Si}, \mathrm{Ge}, R_{S i}^{0}$ and $R_{G e}^{0}$ are the bond lengths in pure $\mathrm{Si}$ and Ge crystals, and $\Delta_{i j}$ is the slope of the dependence of the average bond length of type $i j$ upon $x$. When $a^{* *}=1$, the lattice is flexible, so every bond adjusts to its natural length (so called Pauling limit), while $a^{* *}=0$ corresponds to a perfectly rigid lattice, so that all bonds adjust their lengths to a common value (Vegard limit) [31].

Table 1. Topological rigidity parameters, calculated in this work (SW and T potentials) and Refs. [27, 31, 32] or measured experimentally [33]

\begin{tabular}{|c|c|c|c|c|c|c|}
\hline \multirow{2}{*}{ Bonds } & \multicolumn{6}{|c|}{$a^{*^{*}}$} \\
\cline { 2 - 7 } & TP & SWP & {$[27]$} & {$[31]$} & {$[32]$} & {$[33]$} \\
\hline $\mathrm{Si}-\mathrm{Si}$ & 0.73 & 0.73 & 0.73 & 0.74 & 0.75 & 0.94 \\
\hline $\mathrm{Si}-\mathrm{Ge}$ & 0.64 & 0.63 & 0.69 & 0.73 & 0.75 & 0.84 \\
\hline $\mathrm{Ge}-\mathrm{Ge}$ & 0.50 & 0.62 & 0.65 & 0.71 & 0.78 & 0.70 \\
\hline
\end{tabular}

The values of $a_{i j}^{* *}$ calculated by us using two different potentials (T and SW) and the theoretical and experimental results of several previous works are compiled in Table 1. Our results obtained with two different potentials do not differ considerably (except for Ge-Ge bond) and are most close to those obtained in the ab initio study [27] where the bulk alloy was modeled applying periodical boundary conditions. Comparing to the results of Ref. [31] where the Tersoff potential and periodic boundary conditions were used, we note a considerable difference for $\mathrm{Si}-\mathrm{Ge}$ and $\mathrm{Ge}-\mathrm{Ge}$ bonds. The data 
obtained by X-ray-absorption fine structure measurements [33] indicate even smaller variations of bond lengths with composition in relaxed epitaxially grown alloys, especially for the case of Si-Si bond (nearly Pauling limit). Despite the discrepancies in the values presented in Table 1, we can conclude that in $\mathrm{Si}_{1-x} \mathrm{Ge}_{x} \mathrm{NCs}$, likewise in the bulk alloy, bond lengths depend on composition much weaker than prescribed by Eq. (1) and that these variations are specific for each bond type. The type of boundary conditions (periodic or rigid) applied at the crystallite surface also produces some effect on Si-Ge and Ge-Ge bonds.

\section{Calculated results and discussion}

\subsection{Phonon DS and localization}

The vibration modes of the generated NCs were calculated by diagonalizing the dynamical matrix composed of the second derivatives of either SW or T potential (the same as was used for the NC relaxation) with respect to atomic coordinates, at equilibrium and we kept the same rigid boundary conditions described in Sec. 2.1. The density of states versus frequency was calculated as a sum of Lorentzian functions (with a homogeneous broadening of $10 \mathrm{~cm}^{-1}$ ) centred at each eigenfrequency. Fig. 2 presents the total DS averaged over a number of samples for each composition. These results correspond to the largest crystallites studied (12 atomic shells).

In general, the shape of the phonon DS obtained in our calculations is similar to the previously calculated data for $\mathrm{Si}_{x} \mathrm{Ge}_{1-x}$ alloy $\mathrm{NCs}[18,19]$. In the optical phonon range $\left(\omega / \omega_{\max }>0.5, \omega_{\max }\right.$ is the maximum frequency in the calculated spectrum) we clearly see three bands corresponding to the experimentally observed Raman scattering peaks $\left(\omega / \omega_{\max } \approx 0.6,0.8\right.$ and 1$)$, well known for the bulk alloy. In the range of acoustical phonons we observe more substantial differences between the DS curves obtained with the SW and T potentials. Interestingly, the acoustic phonon DS calculated with the $T$ potential looks quite similar to the experimental [34] and calculated [35] data for hydrogenated amorphous $\mathrm{Si}$, which is another silicon-based disordered system. The feature at $\omega / \omega_{\max } \approx 0.15$ (seen only in the spectra obtained with the $\mathrm{T}$ potential) corresponds to the Brillouin zone edge TA phonons of germanium $\left(\approx 80 \mathrm{~cm}^{-1}\right)$. Some of the smaller features in the middle of the spectrum seem to be associated with surface vibrations but we have not been able to clearly identify their range of localization by inspecting the eigenvectors.

In order to investigate the localization of some characteristic modes, we calculated the inverse participation ratio [29]:

$$
\operatorname{IPR}\left(\omega_{j}\right)=\sum_{l=1}^{N}\left(\mathbf{u}_{l}^{j}\right)^{4} /\left(\sum_{l=1}^{N}\left(\mathbf{u}_{l}^{j}\right)^{2}\right)^{2}
$$

where $\mathbf{u}_{l}^{j}$ is the displacement vector of $l$-th atom for $j$-th vibration mode. Large IPR values are characteristic of strongly localized modes with only few atoms vibrating. Some typical IPR spectra are presented in Fig. 3. As expected, the optical phonon modes are stronger localized than the low-frequency acoustical vibrations. By analyzing the corresponding eigenvectors, we established that the DS peak designated as $\mathrm{Si}$-Ge mode $\left(\omega / \omega_{\max } \approx 0.8\right)$ is associated with vibrations of $\mathrm{Si}$ atoms surrounded 
by three almost motionless Ge atoms. By virtue of this observation, we think that it should not be designated as a stretching mode of Si-Ge bonds. Further support for this statement is provided by Raman spectra discussed in the next section.

\subsection{Raman spectra}

Non-resonant Raman spectra were calculated within the bond polarizability model [36] where three second-rank tensors are defined for each phonon mode $(j)$ :

$$
\begin{gathered}
\vec{\alpha}_{1}=\sum_{l=1}^{N} \sum_{i=1}^{4}\left(\hat{r}_{l i} \otimes \hat{r}_{l i}-\frac{\vec{I}}{3}\right) \mathbf{u}_{l}^{j} \cdot \hat{r}_{l i} ; \quad \vec{\alpha}_{2}=\sum_{l=1}^{N} \sum_{i=1}^{4}\left[\frac{1}{2}\left(\hat{r}_{l i} \otimes \mathrm{u}_{l}^{j}+\mathrm{u}_{l}^{j} \otimes \hat{r}_{l i}\right)-\frac{\vec{I}}{3}\right] \mathbf{u}_{l}^{j} \cdot \hat{r}_{l i}, \\
\vec{\alpha}_{3}=\sum_{l=1}^{N} \sum_{i=1}^{4} \vec{I} \mathbf{u}_{l}^{j} \cdot \hat{r}_{l i}
\end{gathered}
$$

In (4) $\hat{r}_{l i}$ is a unit vector directed from atom $l$ to its neighbour $i$. and $\vec{I}=3 \sum_{i} \hat{r}_{l i} \otimes \hat{r}_{l i} / 4$. The tensor $\vec{\alpha}_{1}$ represents the polarizability modulation due to bond stretching and is responsible e.g. for the Raman peak of pure crystalline $\mathrm{Si}$ at $\approx$ $520 \mathrm{~cm}^{-1}$. The $\vec{\alpha}_{2}$ и $\vec{\alpha}_{3}$ contributions vanish in the case of perfect tetrahedric bonding.

The (polarization-averaged) Raman scattering intensity, normalized by the NC volume was calculated as

$$
I(\omega) \propto \frac{n_{\mathrm{BE}}(\omega)+1}{N \omega} \sum_{j} \frac{\left(\alpha_{1 x y}^{2}+\alpha_{1 x z}^{2}+\alpha_{1 y z}^{2}\right)}{3} \delta\left(\omega-\omega_{j}\right),
$$

where $n_{\mathrm{BE}}(\omega)$ is the Bose-Einstein function. Again, for the computational purposes the $\delta$-function in (5) was replaced by a Lorentzian. Some of the calculated spectra are shown in Fig. 4 (T potential), which are in qualitative agreement with both previously calculated $[18,19]$ and experimental $[6-8,16]$ results for Si-Ge NCs. The shape of the spectra is determined by both small size (confinement) and alloy disorder effects.

The size effect is clearly seen in Fig. 5. For $x=0$, the phonon confinement considerably shifts the Raman peak downwards and the shift with respect to the bulk peak position scales approximately as $D^{-2}$ with the NC size $(D)$, in agreement with the macroscopic model predictions [37] and experimental data [6]. Although the TP and SWP results show a considerable discrepancy, the $D$-dependence is quite similar. As it has been pointed out earlier [38], the lattice dynamics of the smallest NCs are similar to large crystallites, in the sense that the phonon modes still are dispersive, therefore spatial confinement introduces a finite minimal phonon wavevector, $k \propto D^{-1}$, so the optical phonon frequency is shifted downwards. For $\operatorname{Si}_{0.5} \mathrm{Ge}_{0.5}$ alloy NCs, the downward shift for smaller sizes is also seen, although it is much weaker than for pure Si (see Fig. 5a). We found a similar trend for the Ge-Ge mode. The existence of the phonon confinement effect in alloy NCs means that the fundamental ( $\mathrm{Si}-\mathrm{Si}$ and $\mathrm{Ge}-\mathrm{Ge}$ ) modes are sufficiently delocalized, at least in a certain range of $x$. Some twenty years ago there was a discussion in the literature concerning phonon localization in $\mathrm{Al}_{x} \mathrm{Ga}_{1-x} \mathrm{As}$ alloys and it has been concluded that, at least, some phonon modes in this two-modal alloy are propagating and can be described by a wavevector (as an appropriate quantum number) [39]. The quantum size effect observed in our 
calculated data, increasing with the growth of $\mathrm{Si}$ fraction for the $\mathrm{Si}-\mathrm{Si}$ mode, is in accordance with this interpretation. It is worth mentioning that continuum model (starting from bulk-like phonon dispersion curves) has been shown to provide a good qualitative description of the CdS-like and CdSe-like Raman peaks in $\mathrm{CdS}_{x} \mathrm{Se}_{1-x} \mathrm{NCs}$ [16], another confined and disordered system.

The Raman scattering intensity, which is supposed to be simply proportional to the scattering volume ( $\mathrm{NC}$ volume, in our case) within a macroscopic approach [40], grows faster with the NC size (see Fig. 5b, note that the Raman intensity is normalised by the number of atoms in the NC). This effect appears also in microscopic continuous models [37].

We also studied the dependence of the positions and heights of the main Raman peaks upon the alloy composition (see Fig. 6). As expected, the Ge-Ge mode grows in intensity and becomes narrower with the increase of Ge contents. For the Si-Si mode, the tendency is the opposite. The asymmetry in the behaviour of the Si-Si and Ge-Ge modes, seen in Fig. 6 , is explained by the difference of the atomic masses. The Si-Ge mode can clearly be identified only for $x>0.2$. Its intensity reaches a maximum at $x \approx$ 0.75 and then decreases and vanishes. We found that the amplitudes of the peaks do not fluctuate too much between different realisations of alloy NC with the same $x$ and $D$. The $x$ dependences of the peak heights, $H$ (right column of Fig. 6) can be approximated by polynomial functions. It has been suggested intuitively that $H$ should scale with the corresponding bond fraction, i.e. according to $(1-x)^{2}$, $2 x(1-x)$ and $x^{2}$ for the Si-Si, Si-Ge and Ge-Ge bonds, respectively [41], A slightly more elaborate formula based on the same idea was proposed in [7]. We found that a reasonable fit can be achieved with cubic polynomials (in arbitrary units):

$$
\begin{aligned}
& H_{S i-S i}=-4.4 x^{3}+14.8 x^{2}-16.9 x+6.5, \\
& H_{S i-G e}=-22.3 x^{3}+34.1 x^{2}-12.4 x+1.7, \\
& H_{G e-G e}=50.8 x^{3}-63.7 x^{2}+27.2 x-3.5,
\end{aligned}
$$

which are considerably different from the above quadratic functions. However, we do not think that the fitting functions (6) have any real physical meaning. The idea that the peak intensity is proportional to the fraction of corresponding nearest neighbour bonds merely fails in the case of $\mathrm{Si}-\mathrm{Ge}$ band (the maximum appears at $x \approx 0.75$ instead of 0.5). It confirms our statement in the end of Sec. 3.1 concerning the origin of this mode, which is related not to a single $\mathrm{Si}-\mathrm{Ge}$ bond but rather to a chain of such bonds. According to the percolation idea [15], the onset of the Si-Ge mode should take place at the threshold composition $x \approx 0.25$. We found that a good fit to the $H_{S i-G e}(x)$ dependence [better than the polynomial fit (6)] can be achieved with the function:

$$
H_{S i-G e}=9.726 \cdot(x-0.15)^{1.558}(1-x)^{0.609},
$$

implying a critical behavior near the Ge fraction of 0.15 . This threshold may correspond to the formation of $\mathrm{Si}-\mathrm{Ge}-\mathrm{Si}-\mathrm{Ge} . .$. chains in the diamond lattice, corresponding to percolating clusters of $\mathrm{Si}$ and $\mathrm{Ge}$ atoms on one of the two $f c c$ sublattices [15]. In fact, the value of 0.15 is close to the known percolation thresholds in the $f c c$ lattice [42]. The percolation idea has clearly been insightful for two-modal pseudo-binary alloys such $\mathrm{Zn}_{1-x} \mathrm{Be} x \mathrm{Se}$, where it concerns the two fundamental optical 
phonon modes [43]. We notice that the intensity of the Ge-Ge mode becomes noticeable only above $x=0.2$ and grows as $H_{G e-G e} \propto(x-0.2)^{\lambda}(\lambda \approx 2.5)$, also implying a percolation-type behavior, although the value of 0.2 is not close to the known percolation thresholds for the diamond lattice [42].

Concerning the dependence of the peak positions on $x$ presented in the left column of Fig. 6, generally speaking, it is in qualitative agreement with the experimental data for $\mathrm{Si}_{1-x} \mathrm{Ge}_{x}$ bulk samples and relaxed films [13-15]. For the largest NC size considered here, the phonon confinement effect is quite small. In fact, the position of the (less localised) Si-Si mode for different $x$ practically coincides with the data of Refs. [13$15]$ and can be approximated by a linear function, $\omega_{S i-S i}=520-65.0 x\left[\mathrm{~cm}^{-1}\right]$. For the Ge-Ge mode (as mentioned above, detectable only for $x>0.2$ ), our results also yield an approximately linear dependence of the peak position on $x$, $\omega_{G e-G e}=298+6.6 x\left[\mathrm{~cm}^{-1}\right]$, which is slightly weaker than obtained in Ref. [14]. It should be pointed out that the precise determination of the position of this mode is rather hard because of its low intensity (for low and intermediate Ge contents), which probably also applies to experiments. This is also true for the Si-Ge mode, for which we obtain frequencies that are somewhat lower than in Ref. [14]. It converges to the Si local vibrational mode $\left(385 \mathrm{~cm}^{-1}[15]\right)$ in the limit $x \rightarrow 1$. In practical terms, the peak positions can be used for the determination of the NC composition and it is better to use the $\mathrm{Si}-\mathrm{Si}$ mode for this purpose. For small NCs where phonon confinement effects can be present, the recipe can be to use the difference between mode positions, $\Delta(x)=\omega_{S i-S i}-\omega_{G e-G e}$, as it has been suggested for other alloy NCs [16].

Smaller features that are clearly seen in the spectra of NCs with intermediate values of $x$ (for both TP and SWP) have also been observed in previous studies, both experimental and computational, of bulk crystals and relaxed epilayers of $\mathrm{Si}_{x} \mathrm{Ge}_{1-x}$ alloys, and their assignment has attracted considerable attention (see [15] and references therein). The three minor peaks marked by arrows in Fig, 4 were observed by Alonso and Winer [13], together with the three major peaks, giving rise to a "sixoscillator model" $[1 \times(\mathrm{Ge}-\mathrm{Ge}), 1 \times(\mathrm{Si}-\mathrm{Ge}), 4 \times(\mathrm{Si}-\mathrm{Si})]$ of the $\mathrm{Si}_{x} \mathrm{Ge}_{1-x}$ Raman pattern. In the recent work [15], it was suggested to consider 7 oscillators, $[1 \times(\mathrm{Ge}-\mathrm{Ge}), 4 \times(\mathrm{Si}-$ $\mathrm{Ge}), 2 \times(\mathrm{Si}-\mathrm{Si})]$. This terminology refers to the early Random Element Isodisplacement and Cluster Isodisplacement models [44] where each characteristic vibration mode was associated with a particular type of atomic cluster (or crystal unit) retaining the lattice symmetry (e.g. a tetrahedron with 4 atoms in the vertices and one in the center). In our calculated results, we have not been able to uniquely associate the minor Raman peaks to some particular alloy configurations of this type. Moreover, the positions of these peaks slightly change for different atomic alloy configurations with the same $x$ and $D$. This is because each Raman peak usually originates from several interconnected crystal units, not a single one [45], and such an assignment may not always be possible. As far as experiments performed on $\mathrm{Si}_{x} \mathrm{Ge}_{1-x}$ NCs are concerned, only one minor mode $\left(\right.$ at $\approx 430 \mathrm{~cm}^{-1}$ ) has been observed so far [8], while e.g. the spectra presented in [3] are clearly free from any extra features.

\subsection{Non-random alloy NCs}

Non-random (i.e. correlated) distribution of atoms in $\mathrm{Si}_{1-x} \mathrm{Ge}_{x}$ alloys was suggested to occur in a number of previous works, even though it seems to be well established that it is thermodynamically stable at (and above) room temperature and undergoes phase separation only below the critical temperature of 170-200 K [31]. Alloy ordering or 
clustering of like atoms are known to produce significant changes in the vibration spectra [29]. In the 80-s, it was suggested that strained epilayers can develop a longrange ordering at $x=0.5$ [46]. Although this idea did not receive further support $[13,15]$, some recent lattice dynamics calculations explored it for NCs predicting a significant red shift of the Si-Ge mode because of the ordering [21]. Our calculations do not confirm this result. For both T and SW potentials we found that the position of the $\mathrm{Si}-\mathrm{Ge}$ mode is the same in completely ordered and random $\mathrm{Si}_{0.5} \mathrm{Ge}_{0.5} \mathrm{NCs}$.

However, experimentally it was observed that separation of $\mathrm{Si}$ and $\mathrm{Ge}$ atoms can take place upon annealing of NCs grown by magnetron sputtering [7,8], eventually leading to the formation of a core-shell structure with a Si-rich $\mathrm{SiGe}$ core surrounded by a Gerich SiGe shell [7].

We investigated the effect of short-range clustering of like atoms by performing the following numerical experiment:

(i) A reference $\mathrm{NC}$ sample (\#1) with $x=0.5$ and random distribution of $\mathrm{Si}$ and $\mathrm{Ge}$ atoms was built and relaxed as before;

(ii) Prior to the relaxation, some Monte Carlo type exchanges of Si atoms completely surrounded by $\mathrm{Ge}$ neighbors (i.e., forming $\mathrm{SiGe}_{4}$ clusters) with $\mathrm{Ge}$ atoms incorporated in $\mathrm{Si}_{4} \mathrm{Ge}$ clusters were performed, yielding $\mathrm{NC}$ samples with different degrees of short-range clustering of like atoms (samples \#2 and 3). The short range clustering parameter was evaluated by counting the fraction of $\mathrm{Si}-\mathrm{Si}$ or $\mathrm{Ge}-\mathrm{Ge}$ pairs as nearest neighbors,

$$
\xi_{S i}=P_{S i-S i}-(1-x)^{2} ; \quad \xi_{G e}=P_{G e-G e}-x^{2}
$$

The calculated Raman spectra of samples \#1-3 are shown in Fig. 7. As expected, the intensity of the $\mathrm{Si}-\mathrm{Ge}$ mode decreases with the increasing degree of clustering. Another clear effect is the blue shift of the Si-Si fundamental modes with clustering, also known for bulk alloys [29]. Apparently, clustering reveals a double-peak structure of the Si-Ge band, however, this effect, as well as the changes in the minor peaks seen in Fig. 7 require further investigation. The acoustic phonon region reveals some low frequency modes that have been observed in nearly spherical NCs of some materials and are explained by the acoustic phonon confinement (so called Lamb's modes, see e.g. [47]). These modes seem to be little affected by the clustering.

\section{Conclusion}

We have shown the potential of our approach using three-particle empirical potentials for modelling the vibrational properties of $\mathrm{Si}_{1-x} \mathrm{Ge}_{x}$ NCs. It has some advantages in comparison with both the $a b$ initio density functional theory approach (the possibility of considering larger crystallites) and the popular empirical VFF model (the incorporation of relaxation to equilibrium structure). Both Stillinger-Weber and Tersoff potentials are suitable, although the latter is more convenient, less timeconsuming and yields results that, for this particular system, are in a better agreement with available experimental data on the variation of bond lengths and phonon-related Raman peaks with NC composition. Our results confirm the trimodal character of the bond length distribution and the importance of the bond bending in $\mathrm{Si}_{1-x} \mathrm{Ge}_{x} \mathrm{NCs}$, similar to the bulk alloys, although some effect of confined geometry is found for GeGe bond. 
We modelled the vibration properties of $\mathrm{Si}_{1-x} \mathrm{Ge}_{x} \mathrm{NCs}$ embedded in a matrix (e.g. $\mathrm{SiO}_{2}$ ) by applying rigid boundary conditions. Extension to free-standing boundary conditions, relevant to e.g. colloidal NCs, is also possible, which can be implemented by saturating dangling bonds of the outmost shell with hydrogen atoms. From macroscopic models one can expect that the eigenfrequencies will change, compared to the rigid boundary conditions' case [48]. Consequently, the shape of the Raman spectrum will be affected but the confinement effect still will be present. An intermediate situation relevant to e.g. Si NCs embedded in a-Si:H matrix, a system of considerable technological interest $[49,50]$, seems to be more complex. Here a weak phonon confinement can be expected, which makes the problem much more sophisticated computationally because it would be necessary to include explicitly a considerable volume of the matrix material or find a reasonable approximation for it. We plan to address this problem in the future. So far, we have shown that the confinement effect is present for the fundamental (Si-Si in Si-reach NCs and Ge-Ge in Ge-rich NCs) modes in the Raman spectra, consisting in the downward shift of the mode frequencies in NCs with very small size. It implies that these phonon modes are dispersive. This effect is clearly seen also $\mathrm{Si}_{0.5} \mathrm{Ge}_{0.5} \mathrm{NCs}$, however, it is much weaker than in monoatomic quantum dots. The intermediate ( $\mathrm{Si}-\mathrm{Ge}$ ) mode is shown to be related to the vibrations of solitary $\mathrm{Si}$ atoms surrounded by three almost motionless Ge atoms. It looks plausible that the intensity of these vibrations increases strongly when the $\mathrm{Si}$ atoms connect into $\mathrm{Si}-\mathrm{Ge}-\mathrm{Si}-\mathrm{Ge} . .$. chains (still surrounded by the majority of Ge atoms), showing a percolation type behavior. The Si-Ge mode is little affected by the confinement effect.

We obtained fitting expressions for the amplitudes of these modes versus composition (6), however, these polynomial fits are essentially cubic and the idea that these amplitudes should be proportional to the fraction of the corresponding bonds, does not seem to work. Instead, we suggest that a fitting function of type (7), characteristic of a critical behaviour, can be more insightful for the Si-Ge and $\mathrm{Ge}-\mathrm{Ge}$ modes. We also found fitting expressions for the fundamental Raman peak positions that may be used for the determination of alloy composition in NCs. However, it is necessary to bear in mind that both can be affected by (i) phonon confinement and (ii) short-range clustering effects. Short-range clustering enhances the $\mathrm{Si}-\mathrm{Si}$ and $\mathrm{Ge}-\mathrm{Ge}$ modes and pushes them upwards in frequency, while it strongly decreases the intensity of the SiGe Raman mode.

\section{Acknowledgments}

Financial support from the Portuguese Foundation or Science and Technology (FCT) and FEDER through Projects PTDC-FIS-113199-2009 and PEst-C/FIS/UI0607/2013 is gratefully acknowledged.

\section{Bibliography}

[1] W. D. A. M. de Boer et al., Nature Nanotechnology, 5 (2010) 878.

[2] A. Malachias, S. Kycia, G. Medeiros-Ribeiro, R. Magalhães-Paniago, T. I.

Kamins, and R. S. Williams, Phys. Rev. Lett. 91, 176101 (2003).

[3] V. O. Yakhimchuk et al, Semiconductor Physics, Quantum Electronics \& Optoelectronics 7, 456 (2004). 
[4] A. G. Milekhin et al., JETP Letters 81, 33 (2005).

[5] A. I. Yakimov et al., Phys. Rev. B 73, 115333 (2006).

[6] S. Takeoka, K. Toshikiyo, M. Fujii, S. Hayashi, and K. Yamamoto, Phys. Rev. B

61, 15988 (2000).

[7] N. A. P. Mogaddam et. al., J. Appl. Phys. 104, 134309 (2008).

[8] L. Z. Liu et. al., Chem. Commun. 46, 5539 (2010).

[9] X. D. Pi and U. Kortshagen, Nanotechnology 20, 295602 (2009).

[10] E. L. de Oliveira, E. L. Albuquerque, J. S. de Sousa, G. A. Farias, and F. M.

Peeters, J. Phys. Chem. C 116, 4399 (2012).

[11] F. Erogbogbo, T. Liu, N. Ramadurai, P. Tuccarione, L. Lai, M. T. Swihart, and P. N. Prasad, ACS Nano. 5, 7950 (2011)..

[12] G. Gouadec and P. Colomban, J. Raman Spectrosc. 38 (2007) 598.

[13] M. I. Alonso and K. Winer, Phys. Rev. B 39, 10056 (1989).

[14] V. A. Volodin, M. D. Efremov, A. S. Deryabin, and L. V. Sokolov,

Semiconductors 40, 1314 (2006).

[15] O. Pages, J. Souhabi, V. J. B. Torres, A. V. Postnikov, and K. C. Rustagi, Phys. Rev. B 86, 045201 (2012).

[16] E. S. Freitas Neto et. al., J. Raman Spectrosc. 42, 1660 (2011).

[17] L. Z. Liu et. al., Appl. Phys. A 103, 361 (2011).

[18] S.-F. Ren, W. Cheng, and P. Y. Yu, Phys. Rev. B. 69, 235327 (2004).

[19] W. Cheng, D. Marx, and S.-F. Ren, Front. Phys. China 3, 165 (2008).

[20] A. D. Zdetsis, C. S. Garoufalis, and E. N. Koukaras, J. Math. Chem. 46, 942 (2009).

[21] M. A. Abdulsattar, J. Appl. Phys. 111, 044306 (2012).

[22] S. De Gironcoli, Phys. Rev. B 46, 2412 (1992).

[23] M. Franz, K. F. Dombrowski, H. Rucker, B. Dietrich, K. Pressel, A. Barz, U.

Kerat, P. Dold, and K .W. Benz, Phys. Rev. B 59, 10614 (1999).

[24] J. Tersoff, Phys. Rev. B 395566 (1988).

[25] A. S. Vasin, O. V. Vikhrova, and M. I. Vasilevskiy, Phys. Status Solidi C 10, 701 (2013).

[26] F. H. Stillinger and T. A. Weber, Phys. Rev. B 31 (1985) 5262

[27] P. Venezuela, G. M. Dalpian, and J. R. da Silva, Phys. Rev. B 64, 193202 (2001).

[28] T. Hori, T. Shiga, and J. Shiomi, J. Appl. Phys. 113, 203514 (2013).

[29] M. I. Vasilevskiy, O. V. Vikhrova, and S. N. Ershov, Phys. Solid State 45, 1154 (2003).

[30] L. Verlet, Phys. Rev. 159, 98 (1967).

[31] C. Tzoumanekas and P. C. Kelires, Phys. Rev. B 66, 195209 (2002).

[32] M. Yu, C. S. Jayanthi, D. A. Drabold, and S. Y. Wu, Phys. Rev. B 64, 165205 (2001).

[33] J. C. Aubry, T. Tiliszczak, A.P. Hitchcock, J.-M. Baribeau, and T. E. Jackman, Phys. Rev. B 59, 12872 (1999).

[34] J. S. Custer, M. O. Thompson, D. C. Jacobson, J. M. Poate, S. Roorda, W. C.

Sinke, and F. Spaensen, Appl. Phys. Lett. 64, 437 (1994).

[35] R. M. Ribeiro, V. J. B. Torres, M. I. Vasilevskiy, A. Barros, and P. R. Briddon, Phys. Status Solidi C 7, 1432 (2010).

[36] R. Alben, D. Weaire, J. E. Smith, and M. H. Brodsky, Phys. Rev. B 11, 2271 (1975).

[37] A. G. Rolo and M. I Vasilevskiy, J. Raman Spectrosc. 38, 618 (2007).

[38] A. G. Rolo, M. I. Vasilevskiy, N. P. Gaponik, A. L. Rogach, and M. J. M.

Gomes, Phys. Status Solidi B 229, 433 (2002). 
[39] S. Baroni, S. de Gironcoli, P. Giannozzi, Phys. Rev. Lett. 65, 84 (1990).

[40] W. Hayes and R. Loudon, Scattering of Light by Crystals, Dover Publ., N-Y, 2004.

[41] F. Pezzoli et al., Mater. Sci. Eng. B 124-125, 127 (2005).

[42] B. I. Shklovskii and A. L. Efros, Electronic properties of doped semiconductors, Springer, Heidelberg, 1984 .

[43] G. K. Pradhan et. al., Phys. Rev. B 81, 115207 (2010).

[44] D. W. Taylor, In: Optical Properties of Mixed Crystals, eds. R. J. Elliott and I. P. Ipatova, North Holland Publ., Amsterdam, 1988, pp. 35-131.

[45] C. H. Grein and M. Cardona, Phys. Rev. B 45, 8328 (1992).

[46] D. J. Lockwood, K. Rajan, E. W. Fenton, J.-M. Baribeau, M. W. Denhoff, Solid State Commun. 45, 8328 (1992).

[47] H. Lamb, Proc. London Math. Soc. 13, 189 (1881).

[48] M. I. Vasilevskiy, Phys. Rev. B 66, 195326 (2002).

[49] E. Vallat-Sauvain, C. Droz, F. Meillaud, J. Bailat, A. Shah, and C. Ballif, J. NonCrystalline Solids 352, 1200 (2006).

[50] B. Rezek, E. Sipek, M. Ledinsky, J. Stuchlik, A. Vetushka, and J. Kocka, Nanotechnology 20, 045302 (2009). 


\section{Figure captions}

Fig. 1 Variations of bond lengths with Ge fraction in relaxed $\mathrm{Si}_{x} \mathrm{Ge}_{1-x} \mathrm{NCs}$ calculated using Tersoff (a) and Stillinger-Weber (b) potentials. Squares denote Si-Si, circles Si$\mathrm{Ge}$, and triangles Ge-Ge bond length. Straight lines correspond to Vegard's law.

Fig. 2 Phonon DS calculated for NCs of three different compositions using either Tersoff or Stillinger-Weber potentials as indicated on the plots.

Fig. 3 Inverse Participation Ratio of vibrational modes plotted against their frequencies for three different compositions of $\mathrm{Si}_{1-x} \mathrm{Ge}_{x} \mathrm{NCs}$.

Fig. 4 Raman spectra of $\mathrm{Si}_{1-x} \mathrm{Ge}_{x}$ NCs of five different compositions calculated using Tersoff or Stillinger-Weber potentials. NC size is $3.9 \mathrm{~nm}$. The arrows indicate second order features discussed in the text.

Fig. 5 Position (upper panel) and height (lower panel) of the Si-Si Raman peak versus size for pure silicon $(x=0)$ and $\mathrm{Si}_{0.5} \mathrm{Ge}_{0.5} \mathrm{NCs}$ calculated with either TP or SWP as indicated.

Fig. 6 Positions (left column) and heights (right xolumn) of the main Raman peaks versus $\mathrm{Ge}$ contents for $\mathrm{Si}_{1-x} \mathrm{Ge}_{x} \mathrm{NCs}$ calculated using Tersoff potential. NC size is 3.9 $\mathrm{nm}$.

Fig. 7 Raman spectra of $\mathrm{Si}_{0.5} \mathrm{Ge}_{0.5}$ NCs with different degree of short-range clustering: $\xi_{S i}=\xi_{G e}=0$ (random), $\xi_{S i}=\xi_{G e} \approx 0.05$ (fraction of Si-Si, Si-Ge and Ge-Ge bonds $0.3 / 0.4 / 0.3)$, and $\xi_{S i}=\xi_{G e} \approx 0.12(0.37 / 0.26 / 0.37)$. The inset shows the acoustic phonon region for the same three NCs. Calculations performed with the Tersoff potentials, NC size is $3.9 \mathrm{~nm}$. 

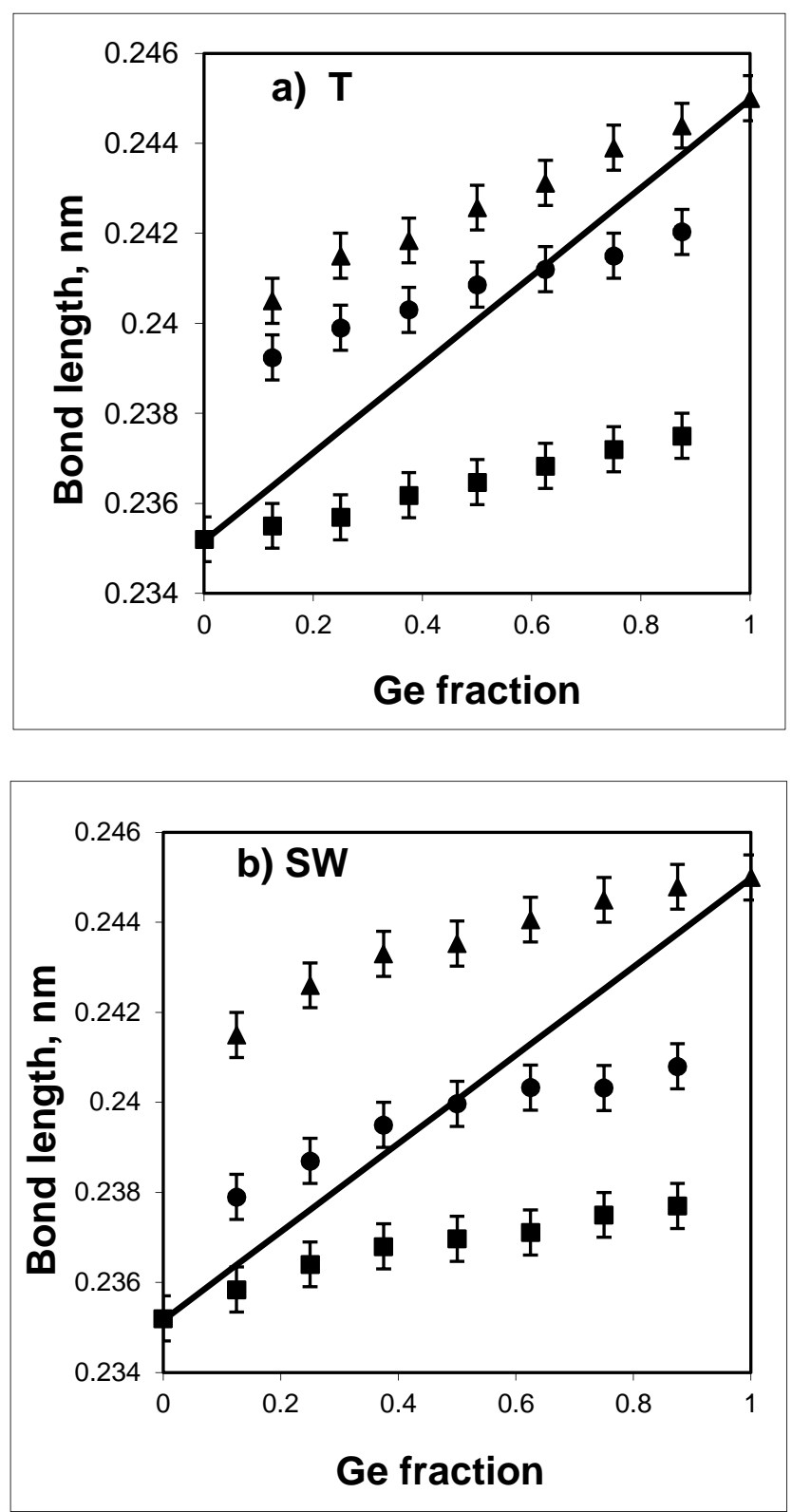


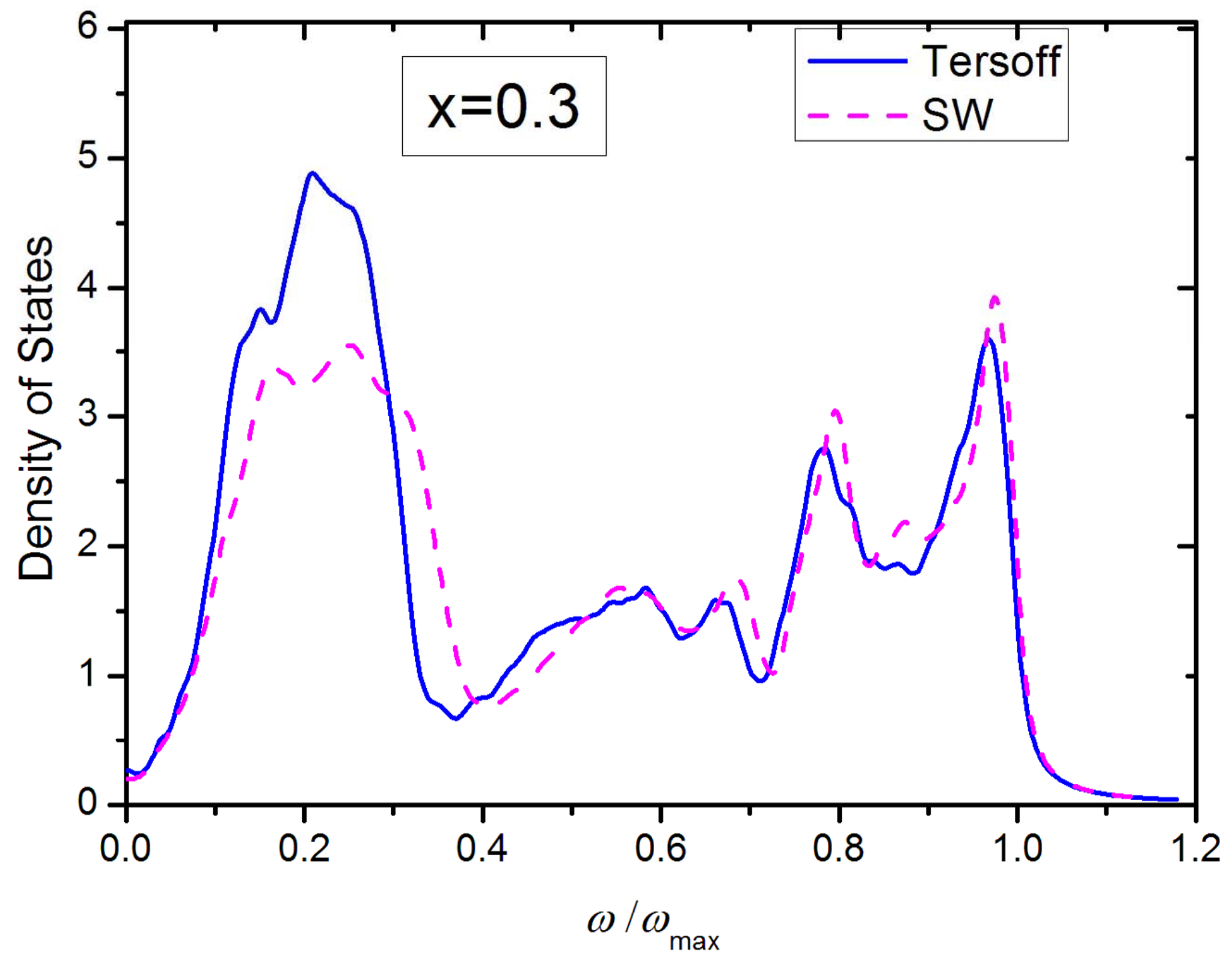




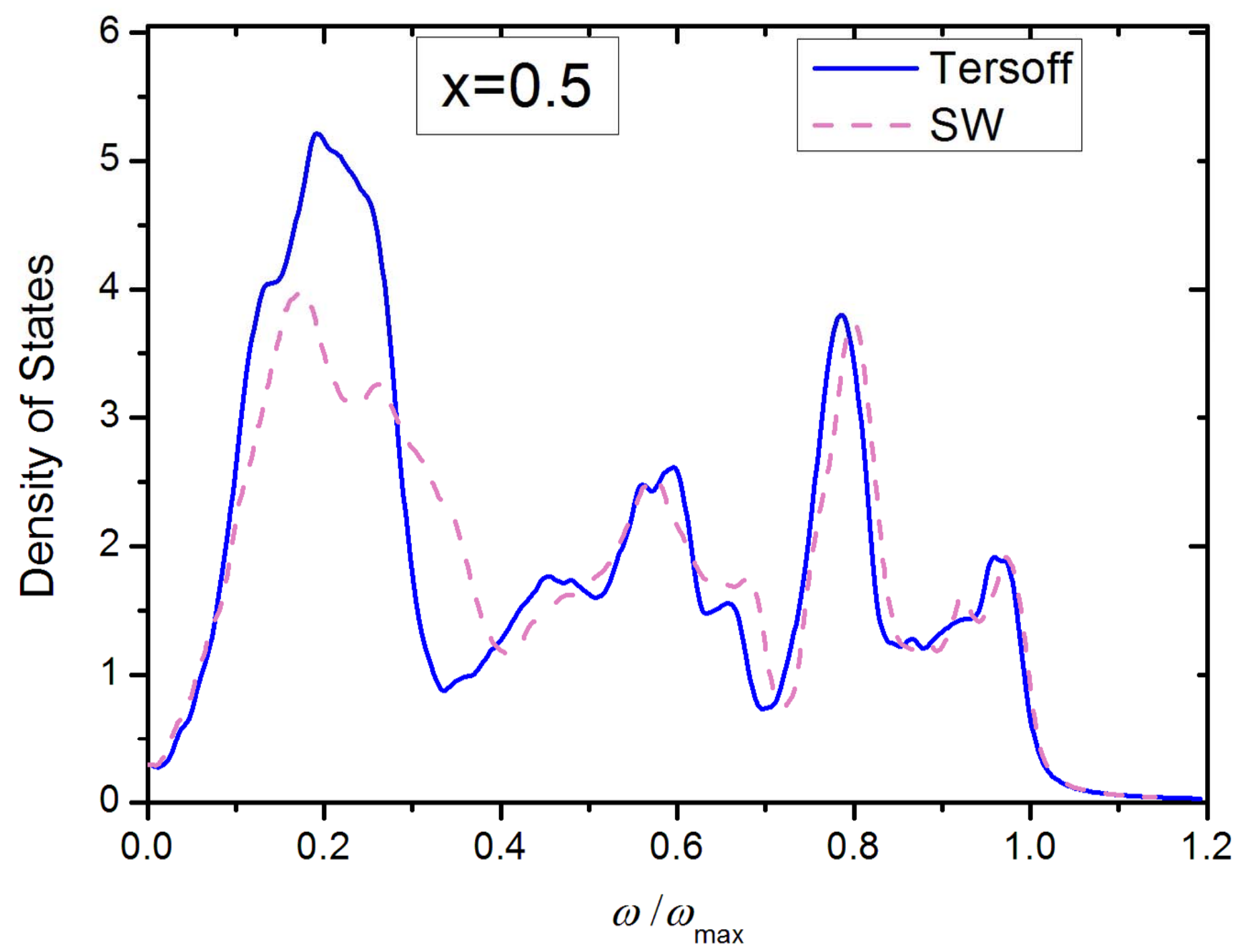




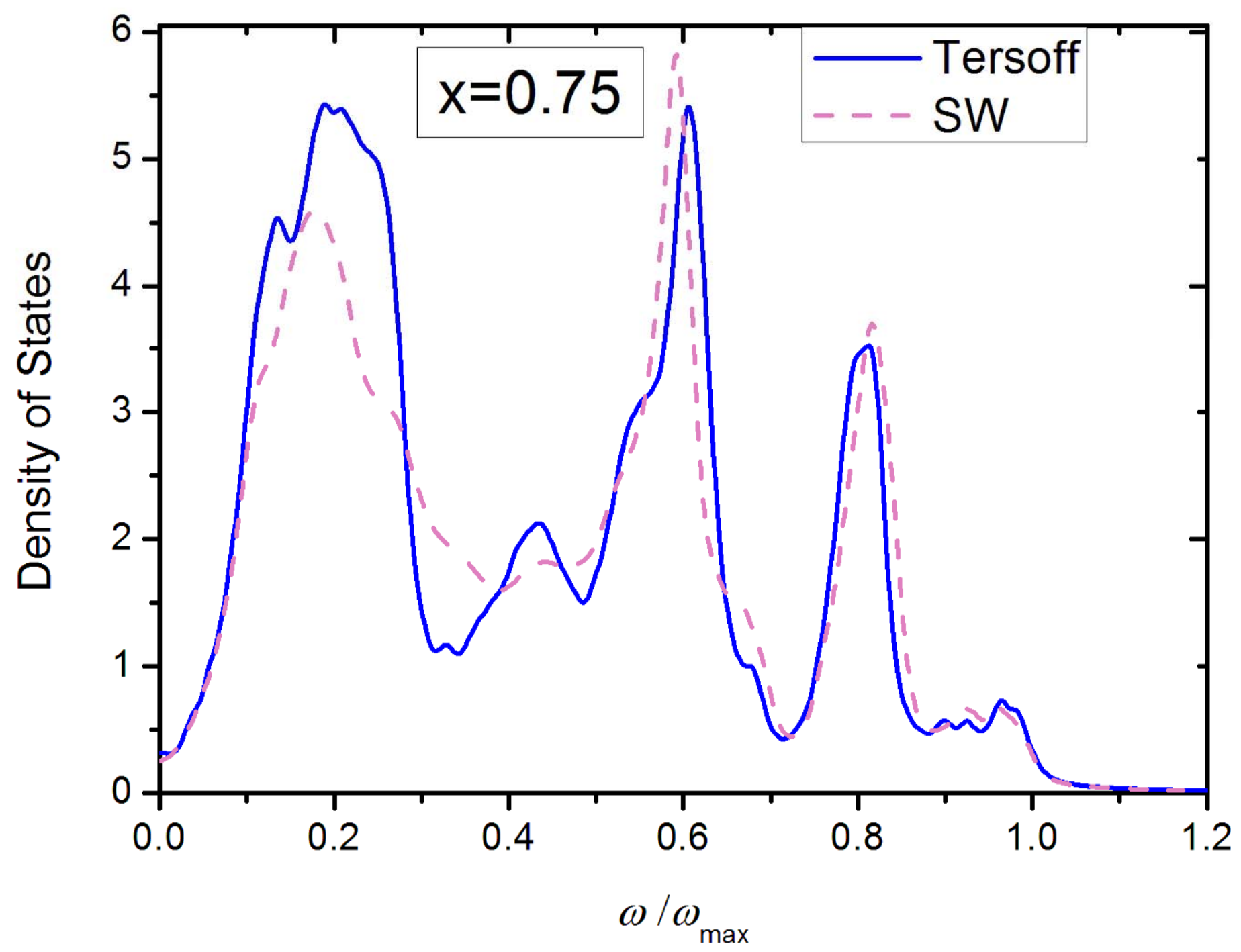




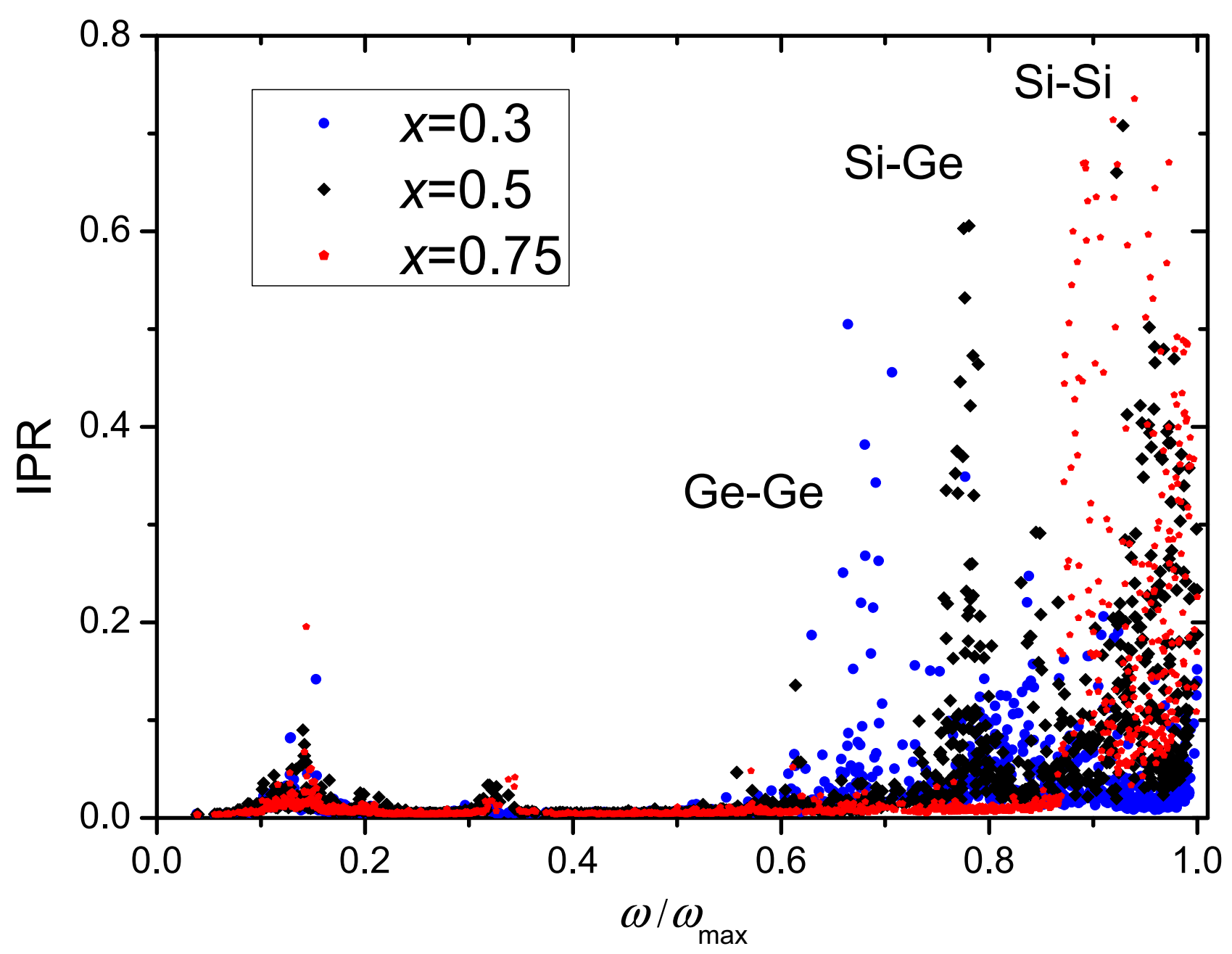




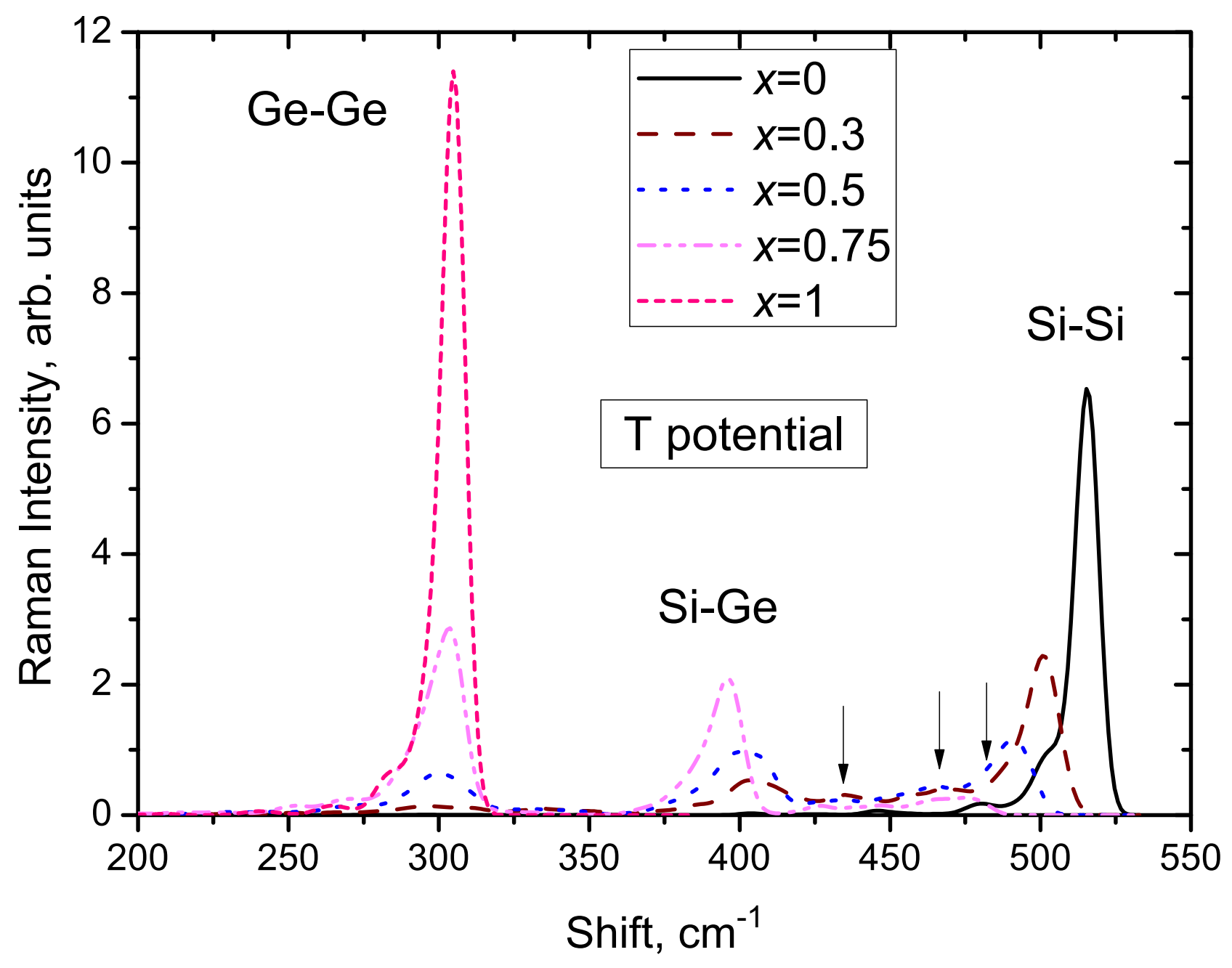




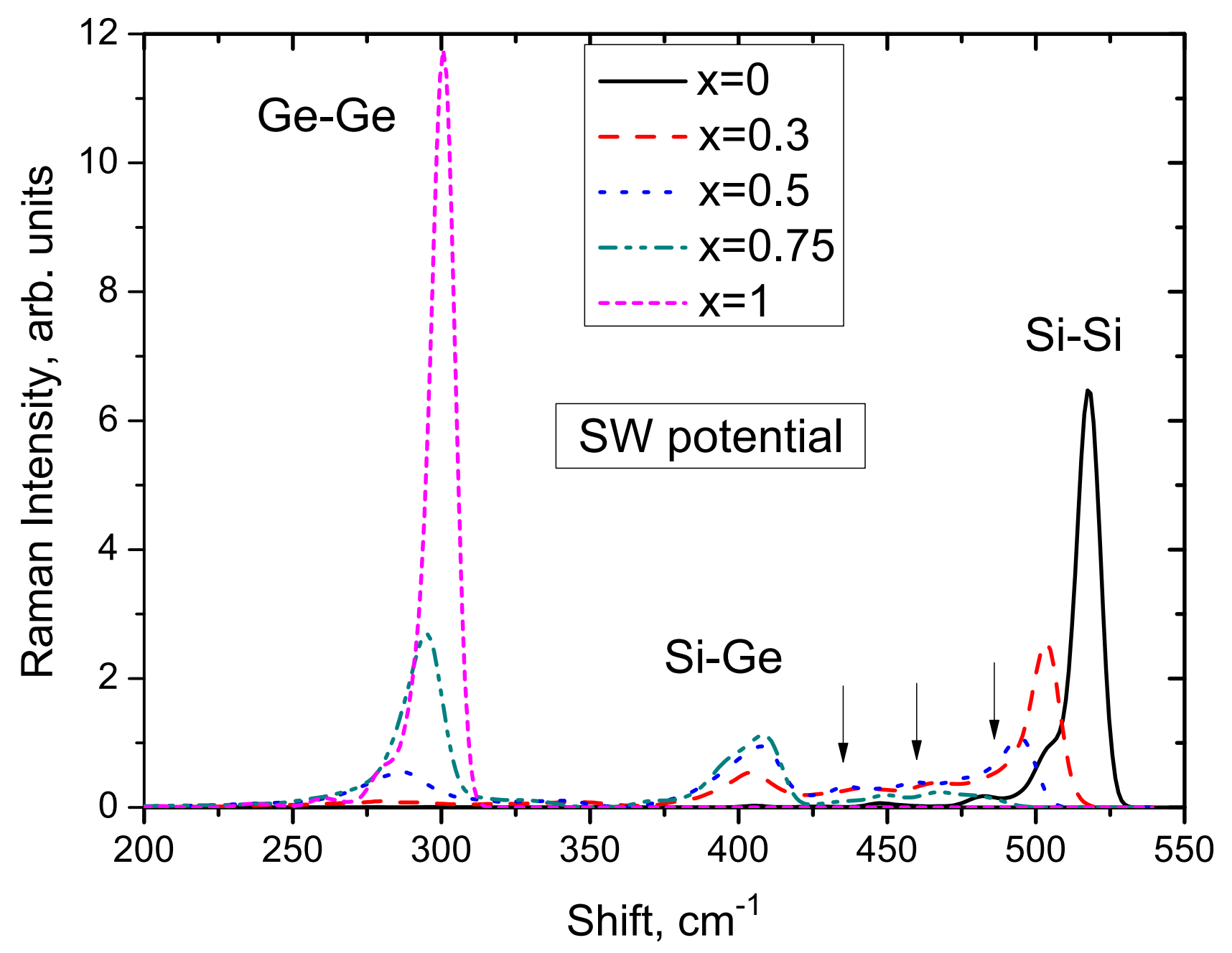




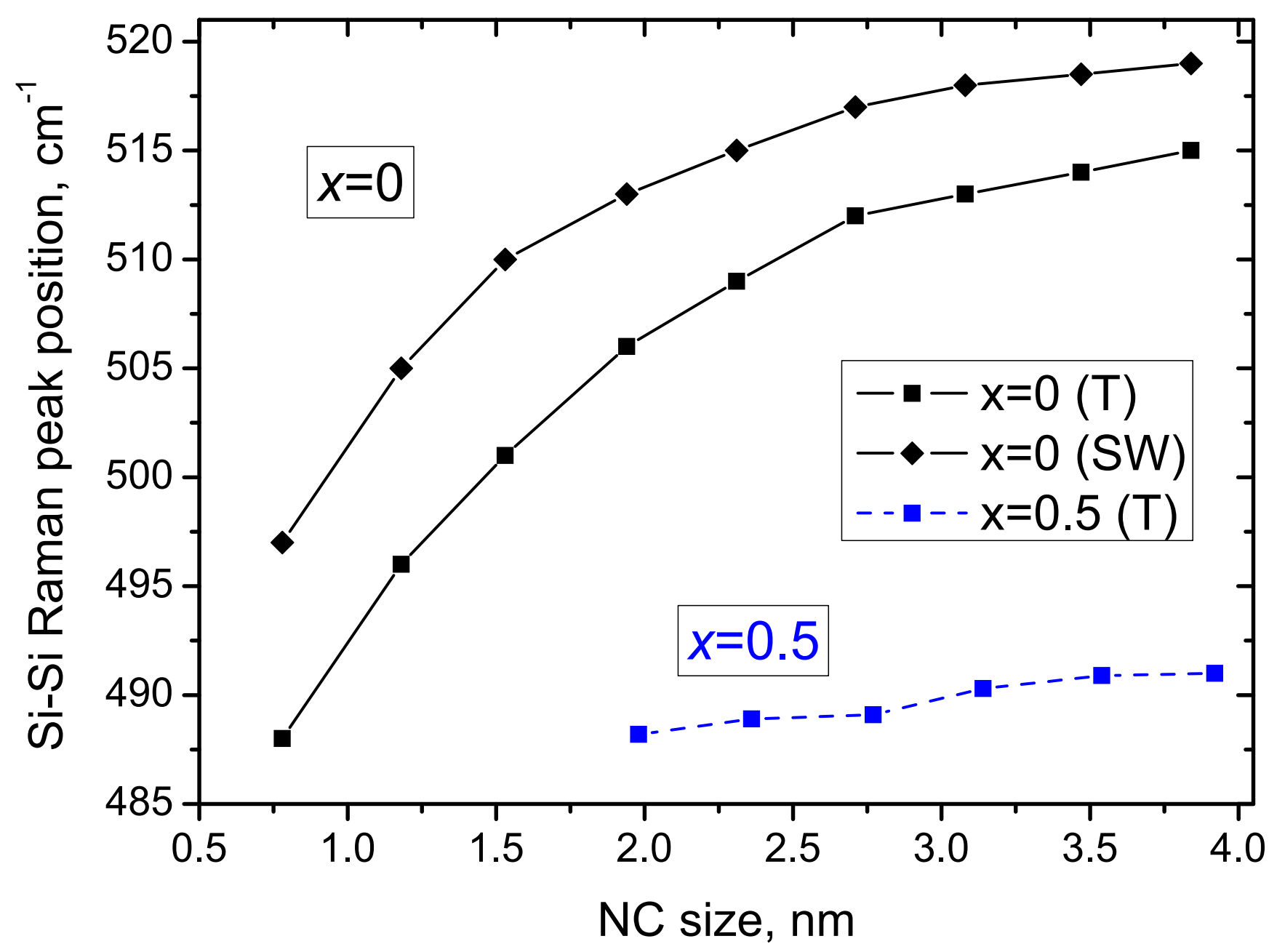




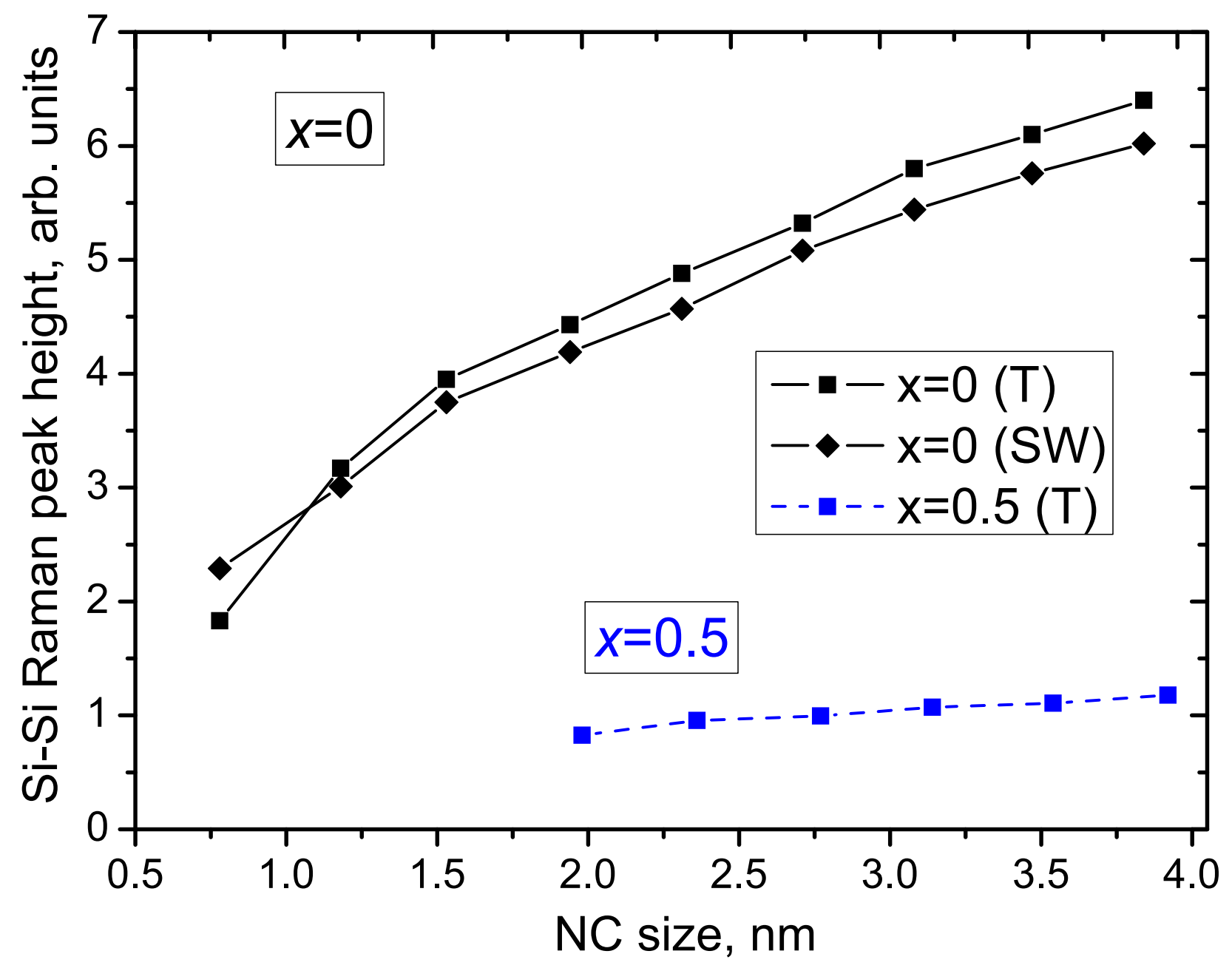



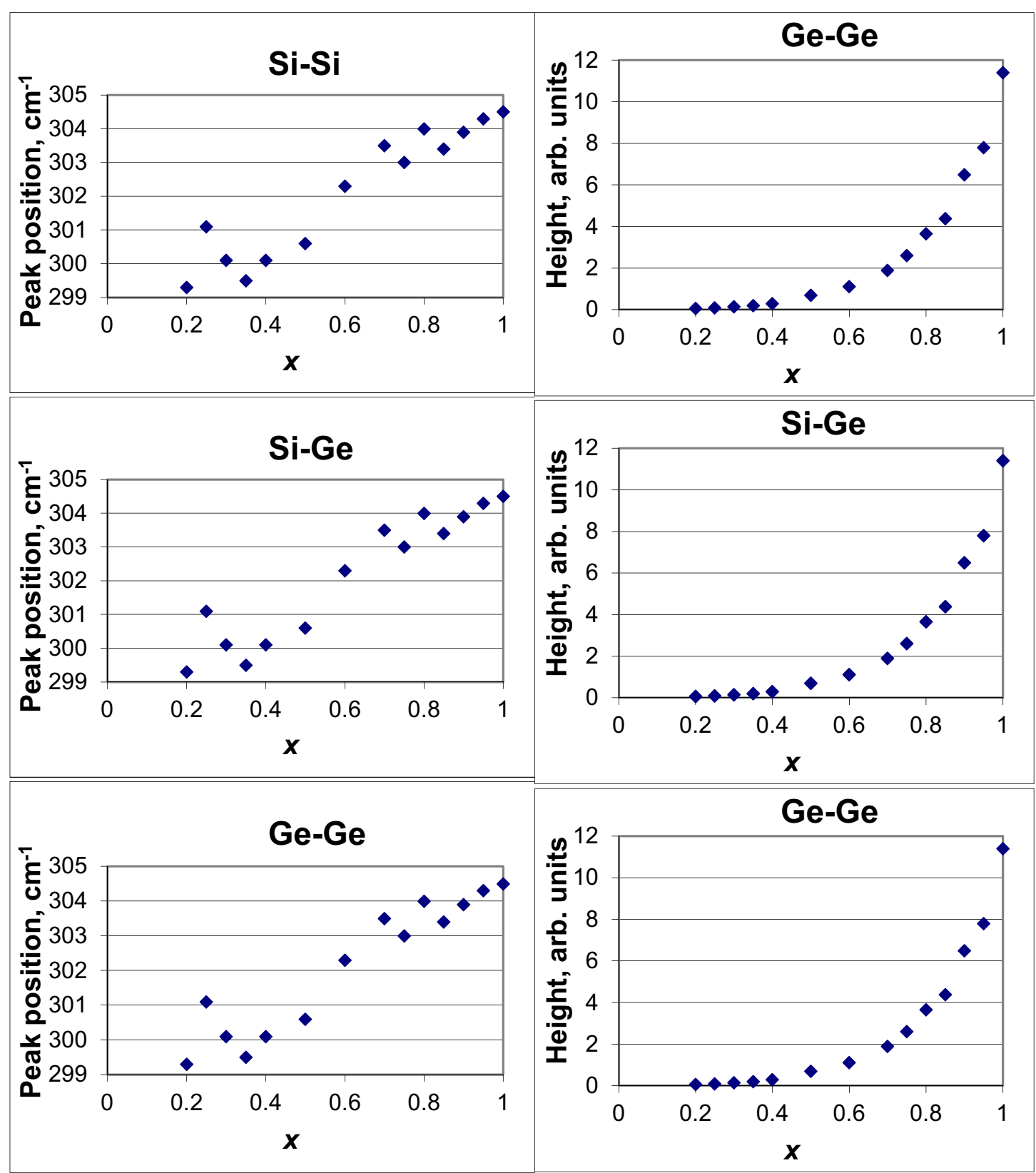


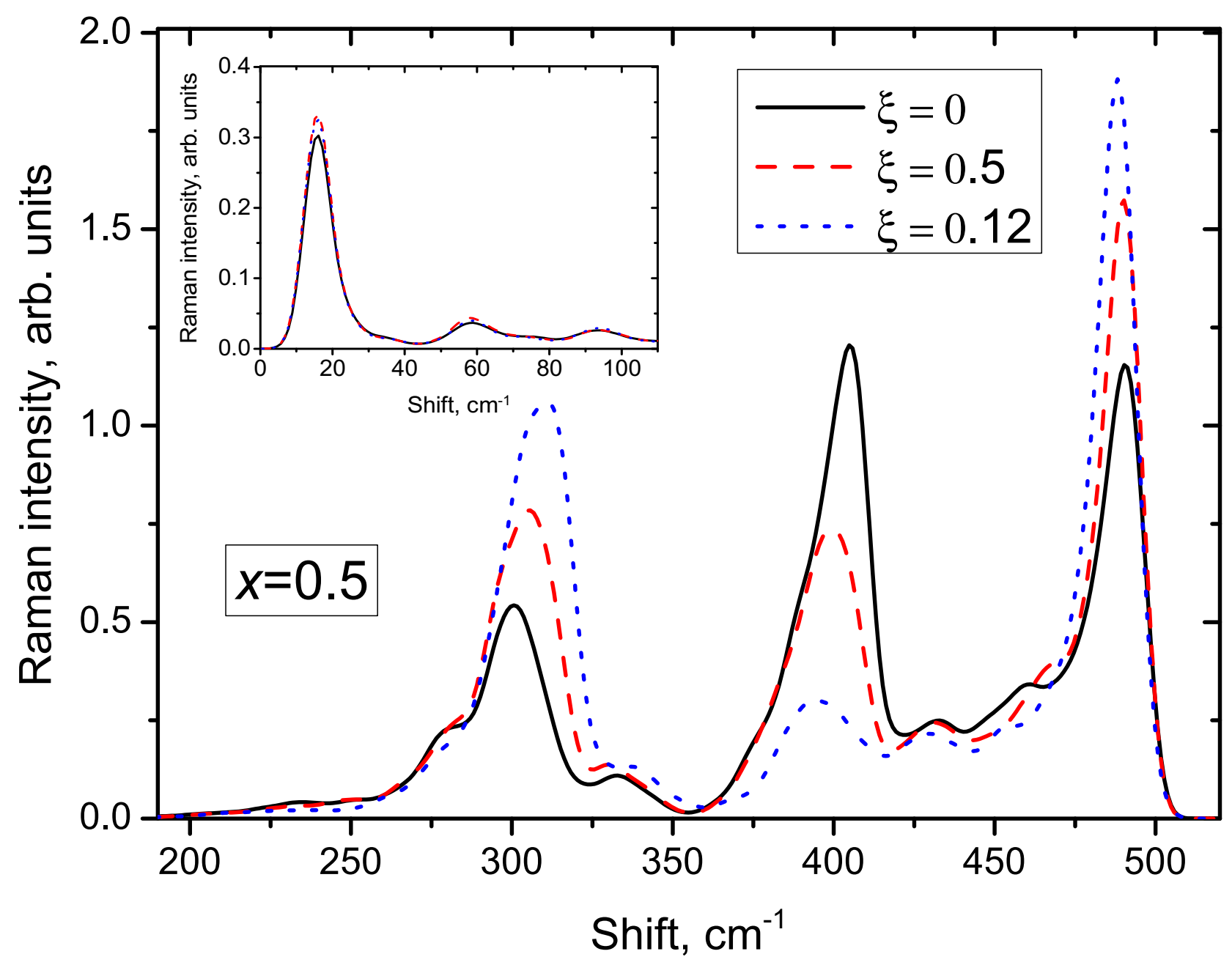

